\title{
Fat Plug Myringoplasty Vs Trichloroacetic Acid Cauterization as Office Procedures for Managing Small Central Perforation
}

\author{
Kanchan Sandeep Dhote, ${ }^{1}$ Priti Rakesh Dhoke, ${ }^{2}$ Vivek Vishwas Harkare, ${ }^{2}$ Nitin Vasant Deosthale, ${ }^{2}$ Sonali Prabhakar \\ Khadakkar $^{2}$
}

\begin{abstract}
Introduction:
In a series of day care office procedures, techniques like fat plug myringoplasty and Trichloroacetic acid (TCA) cauterization are becoming increasingly popular for managing small central perforations now-a-days[Office1]. These are minor, cost effective procedures and have success rates that match success rates of conventional tympanoplasty[Office2]. The aim of this prospective randomized controlled trial, was to compare the effectiveness of fat plug myringoplasty and chemical cauterization in the management of small centrally located perforations of the pars tensa.

\section{Materials and Methods}

The study was carried out over a period of 2 years on 62 patients selected from ENT OPD in our tertiary health care setup of central India. All the selected patients were grouped into two using a random allocation software EPi Info7.1.1.14. Group A included those who underwent fat plug myringoplasty while group B were those who underwent TCA cauterization.

$\underline{\text { Results }}$

At 6 months follow up there was closure of perforation in 29 out of 30(96.6\%) patients of group A and 29 out of 32(91\%) patients of group B. In both groups success was defined by closure of perforation. Comparison of results was done using Fisher Exact Test. The difference between the success rates of the two procedures was statistically insignificant with $p$ value of 0.6624 , indicating that both the office procedures are equally effective and can be used according to the needs of the patients.

\section{Conclusion}

These office procedures can revolutionize the protocol of tympanoplasty which has an obvious higher morbidity and more days of absenteeism as compared to these minor procedures.
\end{abstract}

ABSTRACT

Kevwords:

Myringoplasty; Trichloroacetic Acid; Fat Plug

I

n a developing country like India we encounter a lot of cases of COM. Causes of tympanic membrane perforation include infection, trauma, tympanostomy tube insertion as well as failed surgical repair. Although small sized perforations do not affect hearing too much, they expose the middle ear mucosa to the external

1 - Department of ENT, NKP Salve Institute of medical

Sciences and Research Institute, Nagpur

2 - Department of ENT, NKPSIMS and LMH, Nagpur

\section{Corresponding author:}

Dr Priti Rakesh Dhoke

email: drpritidhoke@rediffmail.com environment leading to repeated troublesome middle ear infections affecting the quality of life of the patients. Hence management of tympanic membrane perforations is required which can be done by repairing the drum surgically.

Small central perforations can be managed by office procedures like fat myringoplasty and chemical cauterization of the rim of perforation.1 Day-care surgeries have now become an integral part of modern Otolaryngology. Different materials have been attempted for closure of perforation like Ivory tube, rubber disc, paper disc etc. Ringenberg ${ }^{2}$ in 1962 first used fat as a graft material for tympanoplasty. In the past people 


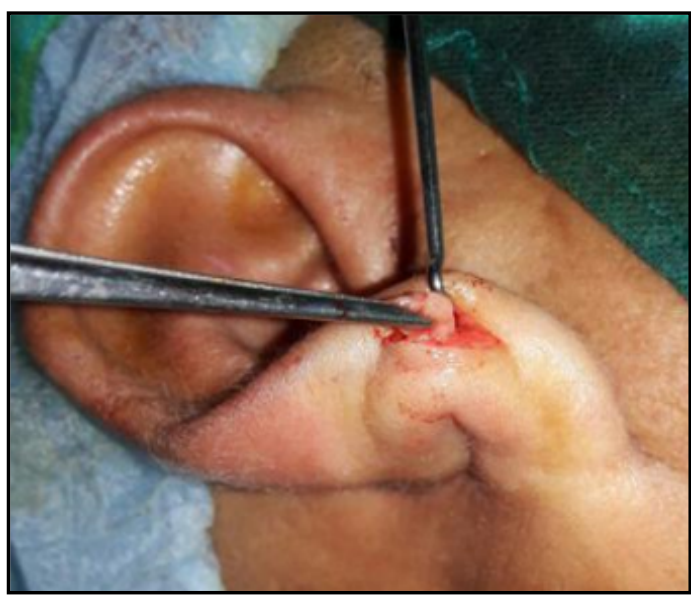

Fig. 1. Harvesting of fat graft from the lobule

used silver nitrate for cauterization of margins of a perforation to promote healing. Okeneff in 1895 started the use of trichloroacetic acid for cauterization which has now become the agent of choice for removal of old epithelial margins.

Tympanoplasty is one of the most common operations performed in otology and temporalis fascia is the most common material used for the closure of tympanic membrane perforation. Conventional tympanoplasty is time consuming, has greater morbidity, is costlier and requires more days of absenteeism as compared to these office procedures. In this study our basic aim was to evaluate the success rates of TCA cauterization and fat plug myringoplasty in the management of small central perforation.

\section{Materials and Methods}

The study was carried out for 2 years from January 2014 to December 2015. The study group included patients in the age group of 16-50 years and having small central perforation in pars tensa which was either following otitis media, trauma or was a post tympanoplasty residual perforation. Those patients who had dry perforations of the size less than $25-30 \%$ of pars tensa in any quadrant, for at least 6 months duration, having mild hearing loss and who agreed for follow up for 6 months postoperatively were included in the study.

Detailed history and clinical examination with otomicroscopy was carried out to assess the perforation

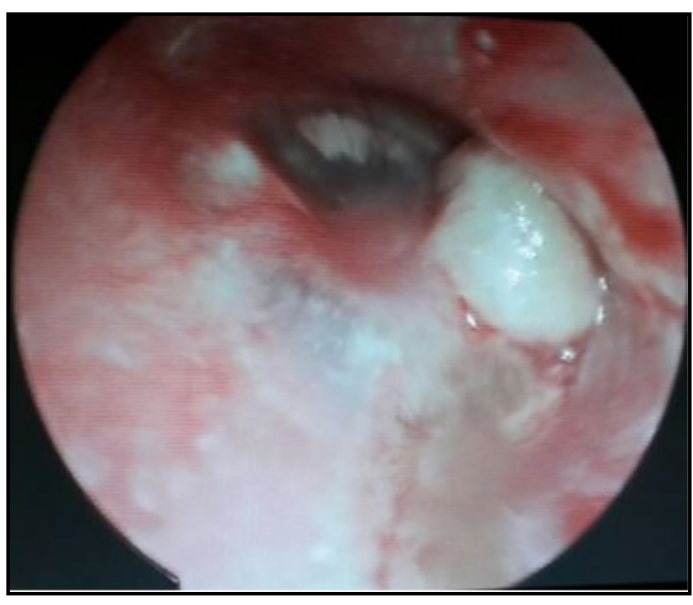

Fig. 2. Inserting fat plug like a dumbbell across the perforation

and to rule out any significant pathology of the tympanic cavity. Preoperative hearing assessment was carried out using tuning forks and confirmed by pure tone audiometry. Nasal and nasopharyngeal pathologies were ruled out in all patients. All the patients who fulfilled the inclusion criteria were divided into 2 groups using random allocation software EPi Info 7.1.1.14. Group A included patients who underwent fat myringoplasty and Group B were those who underwent TCA cauterization. After proper written and informed consent fat plug myringoplasty was performed under local anaesthesia with $2 \%$ lignocaine and adrenaline (1 in 2,00,000) injected around the ear lobule and at four quadrants of the external auditory canal. A $1 \mathrm{~cm}$ vertical incision was made on the posterolateral aspect of the ear lobule. A single fat piece approximately twice the size of the perforation was harvested. (Fig.1)

The wound was then closed with 3-0 silk. The edges of the perforation were de-epithelialized under the operating microscope using sickle knife. The harvested fat plug was then wedged snugly like a dumb bell, one part of which was in the middle ear and other lateral to tympanic membrane, supported by few pieces of antibiotic soaked gel foam on the lateral surface. (Fig. 2)

The patient was started on a course of antibiotic and antihistaminic and topical nasal decongestant for 7 days and then discharged after 2-3 hours on the same day with an instruction to keep the ear dry for 4 weeks. The patient was instructed to avoid straining or nose blowing 


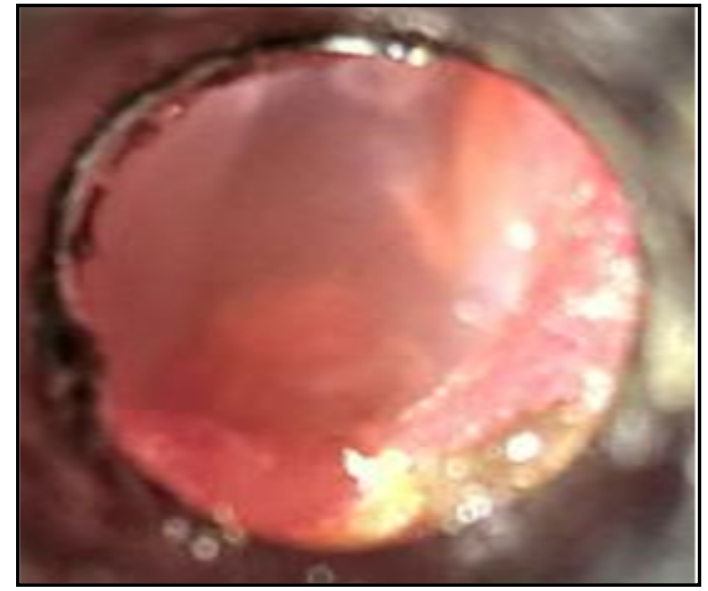

Fig. 3. Hue of fat seen through the healed tympanic membrane

for at least 4 weeks. No pack was kept and the patient was asked to instill local antibiotic drops for 2 weeks in the ear. Patients were followed up regularly for 6 months, biweekly for 1 st month, thereafter at 2-month, 3 months and then 6 months (Fig. 3).

Chemical cauterization was carried out with $50 \%$ Trichloroacetic acid. (Fig. 4) The external auditory canal and tympanic membrane were anaesthetized by placing cotton ball soaked with $4 \%$ lignocaine for 5 mins. Under the operating microscope the edges of perforation were cauterized by cotton wool applicator dipped in trichloroacetic acid solution by stroking in outward direction till a white rim of $0.5 \mathrm{~mm}$ width of cauterized margin was seen.

The patient was then sent home with an instruction to prevent entry of water in the ear and to instill 2-3 drops of ofloxacin ear drop thrice a day. A course of antihistaminic for one week was also given. The patient was then followed up weekly for subsequent sittings. A maximum of 5 sittings were carried out and signs of healing were seen. If a red rim, which is a positive sign of healing, was seen then the procedure was repeated every week till complete healing. If no signs of healing were seen for consecutive 5 sittings then the procedure was abandoned and was subjected to conventional tympanoplasty later. Patients in whom the closure of perforation was achieved were followed up regularly till 6 months.

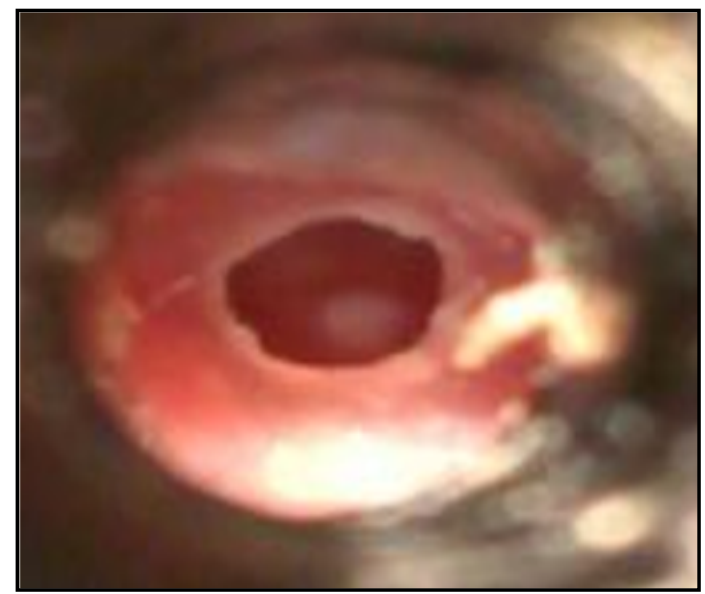

Fig. 4. Cauterized rim of the perforation using Trichloroacetic acid (50\%)

\section{Results}

Among the 62 cases the average age was 29.2 years. In group A there were 17 males and 13 females while in group B there were 10 males and 22 females. The perforations were anterior in 18 patients, posterior in 20 and at the junction of anterior and posterior quadrants in 24 patients (Table I).

In our study 25 cases had minimal conductive hearing loss $(15-25 \mathrm{~dB})$ while 37 cases had mild conductive hearing loss (26- $40 \mathrm{~dB})$. Closure of perforation in patients undergoing fat plug myringoplasty (Group A) was seen in 29 patients while fat got displaced in 1 patient and there was a residual perforation. Closure of perforation in patients undergoing TCA cauterization (Group B) was achieved in 29 patients while 3 patients did not show any signs of improvement even at the end of 5 sittings and hence the procedure was abandoned in them (Table II).

\section{Discussion}

Gone are the days of excessive manipulation of middle ear for small perforations with hardly any hearing loss, inflicting a lot of morbidity to these patients leading to at least 2-3 weeks of absenteeism from work and lot of medications. ${ }^{1,3}$ Minimal interventions in the form of lobular fat graft myringoplasty and trichloroacetic acid cauterization have decreased the agony of patients 
Table I: Site of Perforation

\begin{tabular}{|c|c|c|}
\hline 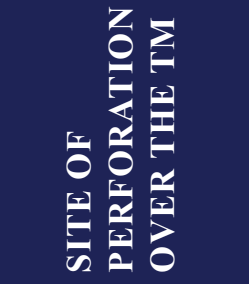 & 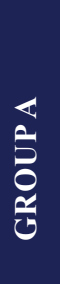 & 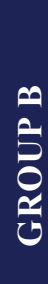 \\
\hline Anterior & 8 & 10 \\
\hline Posterior & 14 & 6 \\
\hline At the junction & 8 & 16 \\
\hline
\end{tabular}

with small perforations, as they are day care procedures requiring minimal hospital stay and are cost effective too. Morbidity is also lesser and the results are comparable to that of conventional tympanoplasty.

Hageman and Hausler ${ }^{4}$ in their study carried out on 44 cases noted closure of perforation after fat plug myringoplasty in $91 \%$ of cases. Chalishagar ${ }^{5}$ described in his series of 20 fat plug myringoplasties that closure of perforation was achieved in $90 \%$ cases at 6 months follow-up. In our study we had $96.67 \%$ success rate at 6 months follow-up with fat plug myringoplasty carried out on 30 patients.

Deddens et al. considered the size of perforation, a crucial factor. Perforations of the size of $5-30 \%$ of the drum surface were considered to have a good prognosis after fat graft myringoplasty, whereas larger perforations were less successful with fat graft alone. ${ }^{6}$ We restricted our study to those patients who had less than or equal to $25-30 \%$ size of perforation $(5 \times 5 \mathrm{~mm})$. It is established that perforations larger than one quadrant of the tympanic membrane are unfavorable for the insertion of fat plug.

There are two theories of histological outcome of fat grafts: Neuhoff's replacement theory ${ }^{7}$ and Peer's cell survival theory. ${ }^{8}$ Transplanted fat according to Nuhof atrophies and is replaced either by fibrous tissue or by newly formed fat which is formed by large wandering histocyte like cell. According to Peer, the fat grafts appeared like normal fat tissue 1 year or more after transplantation. According to him all the original adipose cells do not die. Those fat cells which receive adequate
Table II: Comparison of TM perforation closure in Group A and Group B at the end of 6 months follow up

\begin{tabular}{|c|c|c|}
\hline & Cิ & \\
\hline Successful & $29(96.67 \%)$ & $29(90.62 \%)$ \\
\hline Failure & $1(2.78 \%)$ & $3(9.37 \%)$ \\
\hline Total & 30 & 0 \\
\hline 0 & 30 & 32 \\
\hline
\end{tabular}

Comparison of results by Fisher Exact test was statistically insignificant with $\mathrm{p}$ value of 0.6624

blood supply survive whereas remaining degenerate; this explains the loss of volume. The transplanted fat gets covered by a connective tissue capsule which becomes progressively thinner and provides the basic platform for the grafting of the tympanic membrane.

Fat can be harvested from abdomen, buttock and ear lobule, although the fat obtained from lobule of pinna is easier to harvest, quicker and there is hardly any post operative scar. The fat of the ear lobule is denser and has the ability to be a nidus for epithelial and mucosal tympanic growth. It is characterized by revascularization activity as seen on otoscopy a few days after the procedure. There is a bulge on the tympanic membrane till the fourth month and later the fat graft disappears and a smooth sclerotic area remains by the end of 6 th month. These findings of our study were well in compliance with those of Ozgursoy et al. ${ }^{9}$

The first recorded use of silver nitrate to stimulate closure of tympanic membrane perforations was by William Wilde in $1884^{10}$ The office chemical myringoplasty was introduced by Roosa in 1876 and popularized by Derlacki in 1950 who reported good result and this procedure then came to be known as Derlacki method. ${ }^{11} \mathrm{He}$ along with his colleagues reported $75 \%$ success rates of healing in a study carried out in 131 perforations with an average of 14 treatment sittings requirement. In our series success rate was $90.6 \%$ in treatment of 32 small central perforations with 
an average 6.4 sittings of TCA application. The principle of perforation closure by repeated acid cautery of the rim usually results into normal tympanic membrane with all the five layers. ${ }^{12}$ The principle of chemical cauterization is that it breaks up fibrosis, promotes granulation and new tissue formation at the margins of perforation. According to Goldman, chemical myringoplasty is just not of historical interest but is an effective means of tympanic membrane closure. ${ }^{13}$

\section{Conclusion}

Fat plug myringoplasty and TCA cauterization are both very safe, simple and cost-effective procedures. Both are day care procedures, requiring very short time and have high success rates. Fat myringoplasty can be performed under local anesthesia while TCA cauterization requires topical or no anesthesia. Regenerated tympanic membrane contains all the five layers after both the procedures, while a normally healed tympanic membrane lacks the fibrous layer. The morbidity associated and the days of absenteeism are more with tympanoplasty. In our study the result shows that both the procedures are at par and can serve as suitable alternatives to conventional tympanoplasty for managing small central perforations.

\section{References}

1. Mukherjee M, Paul R. Minimyringoplasty; Repair of small central Perforation of tympanic membrane by Fat Graft: A
Prospective study. Indian J Otolaryngol Head Neck Surg. 2013; 65(4): 302-4

2. Ringenberg JC. Fat graft tympanoplasty. Laryngoscope 1962; 72: $188-92$

3. Kim DK, Park SN, Yeo SW, et al. Clinical efficacy of fat graft myringoplasty for perforations of different sizes and locations. Acta Otolarygol. 2011; 131(1): 22-6

4. Hagemann M, Hausler R. Tympanoplasty with adipose tissue. Laryngorhinootologie 2003; 82(6): 393-6

5. Chalishagar U. Fat plug myringoplasty.Indian J Otolaryngol Head Neck Surg. 2005; 57:43-4

6. Deddens AE, Muntz HR, Lusk RP. Adipose myringoplasty in children. Laryngoscope 1993; 103: 216-9

7. Neuhoff H. The transplantation of tissues, New York; Appleton \& Co. 1923; 74

8. Peer LA. Loss of weight and volume in human fat grafts with postulations of a "cell survival theory." Plast Reconstr Surg. 1950; 5: 217-30

9. Ozgursoy OB, Yorulmaz I. Fat graft myringoplasty: A cost effective but underused procedure. J Laryngol Otol. 2005; 119:277-9

10. Wilde WR, Hewson A. Practical observations on aural surgery and the nature and treatment of diseases of the ear. Blanchard \& Lea, Philadelphia 1853; 292-3

11. Derlacki EL. Office closure of central tympanic membrane perforation: A quarter century experience. Transactions of the American Academy of Ophthalmology 1973; 77: 53-6

12. Shambaugh GE. Glasscock \& Shambaugh Surgery of the Ear. 2nd ed. W.B. Saunders company, Philadelphia 1967; 429-37

13. Goldman NC. Chemical closure of chronic tympanic membrane perforations. ANZ Journal of Surgery 2007; 7(10):850-1. 